\title{
An Exactly Solvable Kondo Problem for Interacting One-Dimensional Fermions
}

\author{
Yupeng Wang ${ }^{1,2}$ and Johannes Voit ${ }^{2}$ \\ 1. Cryogenic Laboratory, Chinese Academy of Sciences, Beijing 100080, P. R. China \\ 2. Physikalisches Institut, Universität Bayreuth, D-95440 Bayreuth, Germany
}

(August 14, 2018)

\begin{abstract}
The single impurity Kondo problem in the one-dimensional (1D) $\delta$-potential Fermi gas is exactly solved for two sets of special coupling constants via Bethe ansatz. It is found that ferromagnetic Kondo screening does occur in one case which confirms the Furusaki-Nagaosa conjecture while in the other case it does not, which we explain in a simple physical picture. The surface energy, the low temperature specific heat and the Pauli susceptibility induced by the impurity and thereby the Kondo temperature are derived explicitly.
\end{abstract}


With the development of nanofabrication techniques for quantum wires and the prediction of edge states in the quantum Hall effect, the interest in 1D electron systems has been renewed in the recent years. Such systems differ fundamentally from those in three dimensions, where the low energy properties can be described very well by the Landau Fermi liquid theory. In the presence of repulsive interaction, quasiparticle excitations are replaced in one dimension by collective excitations. [1,2] The low temperature properties of such systems are then described by the so-called Luttinger liquid theory.

Considerable attention has been focused on the response of a Luttinger liquid to localized perturbations. An example is the exchange interaction between such a non-Fermi liquid and a localized magnetic impurity (Kondo problem in a Luttinger liquid). This problem was first considered by Lee and Toner, [3] who used abelian bosonization to map the problem onto a kink-gas action. The dependence of the Kondo temperature $T_{K}$ on the bare exchange $J$ generically has a power-law dependence on the Kondo coupling $J$ determined by the effective Luttinger coupling $K_{\rho}$, and crosses over to the familiar exponential form for $J$ large or $K_{\rho} \rightarrow 1$. Subsequently, a poor man's scaling treatment on this problem was performed by Furusaki and Nagaosa. [4] They proposed the interesting conjecture that a Luttinger liquid supports a Kondo effect even if the bare exchange interaction is ferromagnetic. Moreover, they showed that the excess specific heat and Pauli susceptibility due to the Kondo impurity are different from those of a local Fermi liquid.

There are a few exact results on the Kondo effect in a Luttinger liquid but no exact solution has been proposed to date. In the absence of electron-electron interaction (Luttinger gas), the problem can be mapped onto a standard 2-channel Kondo problem [5:6] but it is not clear, how these results are changed for a Luttinger liquid. A somewhat artificial model, where the propagation direction of electrons is correlated with their spin direction, does show both ferromagnetic and antiferromagnetic Kondo effects but its thermodynamics is Fermiliquid-like and apparently differs from the Furusaki-Nagaosa solution. [7] Finally, boundary conformal field theory has allowed the classification of all possibilities of critical behavior for a Luttinger liquid coupled to a Kondo impurity. [8] It turns out that there are just two possibilities, a local Fermi liquid with standard low-temperature thermodynamics or a nonFermi liquid with the anomalous scaling observed by Furusaki and Nagaosa. In this letter, we propose an exact solution to two cases of the Kondo problem in a Luttinger liquid. In one case, we find a ferromagnetic Kondo effect similar to Furusaki and Nagaosa [4] but with the thermodynamics of a local Fermi-liquid while in the other case, we do not find complete Kondo screening.

The proper starting point to consider the Kondo problem in a fermion chain is the Hamiltonian

$$
\begin{aligned}
H & =H_{0}+H_{\mathrm{imp}}, \\
H_{0} & =-t \sum_{j=1, \sigma}^{N-1}\left(C_{j \sigma}^{\dagger} C_{j+1 \sigma}+\text { h.c. }\right)+U \sum_{j=1}^{N} n_{j, \uparrow} n_{j, \downarrow}, \\
H_{\mathrm{imp}} & =J \sum_{\sigma, \sigma^{\prime}}\left[C_{1 \sigma}^{\dagger} C_{1 \sigma^{\prime}}+C_{N \sigma}^{\dagger} C_{N \sigma^{\prime}}\right] \tau_{\sigma \sigma^{\prime}} \cdot \mathbf{S}+V \sum_{\sigma}\left[n_{1 \sigma}+n_{N \sigma}\right],
\end{aligned}
$$

where $H_{0}$ is the Hamiltonian of the Hubbard chain with open boundaries and $H_{i m p}$ is the interaction between the electron gas and the local impurity; $\tau$ is the Pauli matrix and $\mathbf{S}$ is 
the local spin- $\frac{1}{2}$ operator. However, even such a simple model can not be solved exactly. To give some exact information of the Kondo effect in a Luttinger liquid, we consider a related continuum model [9]

$$
H=-\sum_{j=1}^{N} \frac{\partial^{2}}{\partial x_{j}^{2}}+2 c \sum_{i<j} \delta\left(x_{i}-x_{j}\right)+\sum_{j=1}^{N}\left[\delta\left(x_{j}\right)+\delta\left(L-x_{j}\right)\right]\left[J \tau_{j} \cdot \mathbf{S}+V\right],
$$

where $c>0$ is the electron-electron coupling constant; $J$ and $V$ describe the Kondo coupling constant and the boundary potential respectively; $L$ is the length of the system and $N$ is the number of electrons. Of course, one would like to solve (1) or (2) with periodic boundary conditions. However, as pointed out earlier [4, 10 impurities in a Luttinger liquid behave always like strong scattering centers at low energy scales and effectively enforce open boundaries.

If a boundary is closed simply by an infinite wall, any electron impinging on this boundary will be completely reflected and suffer a phase shift between incident and reflected waves of $\pi$ [11] 13]. If, however, the wall is preceded by a very narrow potential well, (boundary fields in the lattice models [14,15]), the incident and reflected waves will not be exactly in opposite phase. We therefore specify our model by imposing the boundary conditions that the waves arriving at either end are reflected as

$$
\begin{aligned}
& e^{i k_{j} x} \rightarrow R_{j 0}\left(k_{j}\right) e^{-i k_{j} x}, \quad(x=0), \\
& e^{i k_{j} x} \rightarrow R_{j 0}\left(k_{j}\right) e^{-i k_{j} x-2 i k_{j} L}, \quad(x=L),
\end{aligned}
$$

where $R_{j 0}\left(k_{j}\right)$ is the reflection matrix of the electrons at the boundary. In our case, $R_{j 0}\left(k_{j}\right)$ is an operator rather than a $c$-number because the electron-boundary scattering involves a spin-exchange process between the electrons and the local impurity. The Yang-Baxter equation

$$
S_{j l}\left(k_{j}-k_{l}\right) R_{j 0}\left(k_{j}\right) R_{l 0}\left(k_{l}\right)=R_{l 0}\left(k_{l}\right) R_{j 0}\left(k_{j}\right) S_{j l}\left(k_{j}-k_{l}\right)
$$

constrains the integrability of our model. $S_{j l}\left(k_{j}-k_{l}\right)$ is the electron-electron scattering matrix in the bulk. Manipulating these equations, we find that the Hamiltonian (目) can be solved exactly by including an irrelevant local counter term proportional to $\sum_{j=1}^{N}\left[\delta^{\prime}\left(x_{j}\right)-\right.$ $\delta^{\prime}\left(x_{j}-L\right)$ ] (ensuring that the wave function vanishes outside the domain $0 \leq x \leq L$ ), and for the following sets of parameters: (i) $J=-2 V=-c / 2$; (ii) $J=2 \mathrm{~V} / 3=-c / 2$; (iii) $J=0$. Case (iii) is just the open boundary problem with boundary potentials of the $\delta$-potential Fermi gas model which has been discussed by Woynarovich [16], and we shall not repeat the discussion here. Case (i) corresponds to a repulsive boundary potential which, following Luttinger liquid theory, would not influence the occurrence of the antiferromagnetic Kondo effect [6]. Nothing is known about its influence on the ferromagnetic Kondo effect. Case (ii) represents an attractive boundary potential which has not been considered previously.

The eigenvalue problem of Hamiltonian (2) is similar to those of other integrable models [11 13,16 and gives the following Bethe ansatz equations

$$
\begin{array}{r}
e^{2 i k_{j} L}=s^{2}\left(k_{j}\right) \prod_{\alpha=1}^{M} \frac{k_{j}-\lambda_{\alpha}+i \frac{c}{2}}{k_{j}-\lambda_{\alpha}-i \frac{c}{2}} \frac{k_{j}+\lambda_{\alpha}+i \frac{c}{2}}{k_{j}+\lambda_{\alpha}-i \frac{c}{2}}, \\
\prod_{j=1}^{N} \frac{\lambda_{\alpha}-k_{j}+i \frac{c}{2}}{\lambda_{\alpha}-k_{j}-i \frac{c}{2}} \frac{\lambda_{\alpha}+k_{j}+i \frac{c}{2}}{\lambda_{\alpha}+k_{j}-i \frac{c}{2}}\left(\frac{\lambda_{\alpha}+i \frac{c}{2}}{\lambda_{\alpha}-i \frac{c}{2}}\right)^{2}=\prod_{\beta \neq \alpha} \frac{\lambda_{\alpha}-\lambda_{\beta}+i c}{\lambda_{\alpha}-\lambda_{\beta}-i c} \frac{\lambda_{\alpha}+\lambda_{\beta}+i c}{\lambda_{\alpha}+\lambda_{\beta}-i c},
\end{array}
$$


with the eigenvalue of the Hamiltonian (2) as

$$
E=\sum_{j=1}^{N} k_{j}^{2},
$$

where $k_{j}$ 's and $\lambda_{\alpha}$ 's are the "rapidities" of the charge and spin respectively, $s(k)=1$ for case (i) and $s(k)=\frac{k-i c}{k+i c}$ for case (ii); $M$ is the number of down spins. For convenience, we put $N$ odd in the following discussion. Our main conclusion is that for case (i), the ground state of the system is a spin singlet which supports the Furusaki-Nagaosa conjecture, while for case (ii), the ground state of the system is a spin triplet which indicates that the local spin can not be screened.

Case (i): No bound state exists and the ground state is described by two sets of real parameters $\{k\}$ and $\{\lambda\}$. Define the quantities

$$
\begin{aligned}
& Z_{L}^{c}\left(k_{j}\right)=\frac{1}{\pi}\left\{k_{j}-\frac{1}{2 L} \theta\left(k_{j}, \frac{c}{2}\right)+\frac{1}{2 L} \sum_{\alpha=-M}^{M} \theta\left(k_{j}-\lambda_{\alpha}, \frac{c}{2}\right)\right\} \\
& Z_{L}^{s}\left(\lambda_{\alpha}\right)=\frac{1}{\pi}\left\{\frac{1}{2 L}\left[2 \theta\left(\lambda_{\alpha}, \frac{c}{2}\right)+\theta\left(\lambda_{\alpha}, c\right)\right]+\frac{1}{2 L} \sum_{j=-N}^{N} \theta\left(\lambda_{\alpha}-k_{j}, \frac{c}{2}\right)-\frac{1}{2 L} \sum_{\beta=-M}^{M} \theta\left(\lambda_{\alpha}-\lambda_{\beta}, c\right)\right\}
\end{aligned}
$$

where $\theta(x, c)=2 \tan ^{-1} x / c$ and we have used the reflection symmetry of the Bethe Ansatz to include solutions with negative $k_{-j}=-k_{j}$ and $\lambda_{-\alpha}=-\lambda_{\alpha}$. The Bethe ansatz (5) is solved by $Z_{L}^{c}\left(k_{j}\right)=\frac{I_{j}}{L}$ and $Z_{L}^{s}\left(\lambda_{\alpha}\right)=\frac{J_{\alpha}}{L}$, where $I_{j}$ and $J_{\alpha}$ are nonzero integers. In the ground state, $I_{j}$ and $J_{\alpha}$ must be consecutive integers to minimize the energy. The roots $k_{j}$ and $\lambda_{\alpha}$ become dense in the thermodynamic limit, and we define their densities as

$$
\rho_{L}^{c}(k)=\frac{d Z_{L}^{c}(k)}{d k}, \quad \rho_{L}^{s}(\lambda)=\frac{d Z_{L}^{s}(\lambda)}{d \lambda}
$$

for length $L$. Their finite size corrections are 14, 17

$$
\begin{aligned}
& \rho_{L}^{c}(k)=\rho_{c}(k)+\frac{1}{L} \delta \rho_{c}(k)+O\left(\frac{1}{L^{2}}\right), \\
& \rho_{L}^{s}(\lambda)=\rho_{s}(\lambda)+\frac{1}{L} \delta \rho_{s}(\lambda)+O\left(\frac{1}{L^{2}}\right) .
\end{aligned}
$$

The densities $\rho_{c}(k)$ and $\rho_{s}(\lambda)$ in the infinite system limit follow from the integral equations

$$
\begin{aligned}
& \rho_{c}(k)=\frac{1}{\pi}+\int_{-\Lambda}^{\Lambda} K\left(k-\lambda, \frac{c}{2}\right) \rho_{s}(\lambda) d \lambda \\
& \rho_{s}(\lambda)=\int_{-k_{0}}^{k_{0}} K\left(\lambda-k, \frac{c}{2}\right) \rho_{c}(k) d k-\int_{\Lambda}^{\Lambda} K\left(\lambda-\lambda^{\prime}, c\right) \rho_{s}\left(\lambda^{\prime}\right) d \lambda^{\prime},
\end{aligned}
$$

and the finite-size corrections $\delta \rho_{c}(k), \delta \rho_{s}(\lambda)$ are solutions of

$$
\begin{aligned}
& \delta \rho_{c}(k)=-K\left(k, \frac{c}{2}\right)+\int_{-\Lambda}^{\Lambda} K\left(k-\lambda, \frac{c}{2}\right) \delta \rho_{s}(\lambda) d \lambda \\
& \delta \rho_{s}(\lambda)=2 K\left(\lambda, \frac{c}{2}\right)+K(\lambda, c)+\int_{-k_{0}}^{k_{0}} K\left(\lambda-k, \frac{c}{2}\right) \delta \rho_{c}(k) d k-\int_{-\Lambda}^{\Lambda} K\left(\lambda-\lambda^{\prime}, c\right) \delta \rho_{s}\left(\lambda^{\prime}\right) d \lambda^{\prime}
\end{aligned}
$$


with $K(x, c)=\pi^{-1} c /\left(x^{2}+c^{2}\right) ; k_{0}$ and $\Lambda$ are cutoffs of $k$ and $\lambda$, respectively, for the ground state, and satisfy $\int_{-k_{0}}^{k_{0}} \rho_{L}^{c}(k) d k=(N+1 / 2) / L$ and $\int_{-\Lambda}^{\Lambda} \rho_{L}^{s}(\lambda) d \lambda=(M+1 / 2) / L$. Notice that $\Lambda \rightarrow \infty$ when $L \rightarrow \infty$. Finally, one can also derive similar integral equations for the dressed energies for the finite $\left(\epsilon_{L}^{c}(k), \epsilon_{L}^{s}(\lambda)\right)$ and infinite system [17. Their finite size corrections behave as

$$
\begin{aligned}
& \epsilon_{L}^{c}(k)=\epsilon_{c}(k)+O\left(\frac{1}{L^{2}}\right), \\
& \epsilon_{L}^{s}(\lambda)=\epsilon_{s}(\lambda)+O\left(\frac{1}{L^{2}}\right),
\end{aligned}
$$

where $\epsilon_{c}(k)$ and $\epsilon_{s}(\lambda)$ are the dressed energies for the infinite system.

We now discuss the physical properties of the system by evalutating its thermodynamics. The boundary energy induced by the impurity is

$$
f=\int_{-k_{0}}^{k_{0}} k^{2} \delta \rho_{c}(k) d k .
$$

The magnetization of the ground state in the thermodynamic limit is

$$
M_{s}=\frac{1}{4} \lim _{L \rightarrow \infty}\left\{2 L \int_{-k_{0}}^{k_{0}} \rho_{L}^{c}(k) d k-4 L \int_{-\Lambda}^{\Lambda} \rho_{L}^{s}(\lambda) d \lambda+3\right\}=0,
$$

where a prefactor $1 / 2$ comes from our use of reflection symmetry (cf. above), and the last term is included to cancel the contributions from the holes at $k=0$ and $\lambda=0$ in the distributions $Z_{L}^{c}(k)$ and $Z_{L}^{s}(\lambda)$, respectively, and that of the impurity. We therefore have a complete screening of the Kondo impurity for ferromagnetic exchange, in agreement with the Furusaki-Nagaosa conjecture [4. Our result implies that a repulsive boundary potential is not detrimental to the ferromagnetic Kondo effect - at least up to the magnitude considered here.

We now determine the Kondo contribution to the specific heat and the Kondo temperature. While the thermodynamics of Bethe Ansatz solvable models can be calculated directly, an alternative, both more practical and more physical, is provided by exploiting the picture of a Landau-Luttinger liquid put forward by Carmelo and coworkers [18]. Here, the densities of states of the quasiparticles at the Fermi energy $E_{F}=0$ are

$$
\begin{aligned}
& N_{L}^{c}(0)=\frac{d Z_{L}^{c}\left(k_{0}\right)}{d \epsilon_{L}^{c}\left(k_{0}\right)}=\frac{1}{2 \pi v_{c}}+\frac{1}{L} \frac{\delta \rho_{c}\left(k_{0}\right)}{\epsilon_{c}^{\prime}\left(k_{0}\right)}+O\left(\frac{1}{L^{2}}\right), \\
& N_{L}^{s}(0)=\frac{d Z_{L}^{s}(\infty)}{d \epsilon_{L}^{s}(\infty)}=\frac{1}{2 \pi v_{s}}+\frac{1}{L} \frac{\delta \rho_{s}(\infty)}{\epsilon_{s}^{\prime}(\infty)}+O\left(\frac{1}{L^{2}}\right),
\end{aligned}
$$

where $v_{c}$ and $v_{s}$ are the velocities of the charge and spin fluctuations, respectively. The densities of states directly determine the low temperature specific heat and magnetic susceptibility and, using standard expressions from Fermi liquid theory, we obtain the additional contributions due to the Kondo effect as

$$
\begin{aligned}
\delta C & =\frac{\pi \delta \rho_{c}\left(k_{0}\right)}{3 L v_{c} \rho_{c}\left(k_{0}\right)} T+\frac{\pi \delta \rho_{s}(\infty)}{3 L v_{s} \rho_{s}(\infty)} T, \\
\delta \chi & =\frac{\delta \rho_{s}(\infty)}{L \rho_{s}(\infty)} \chi_{0},
\end{aligned}
$$


where $\chi_{0}$ is the susceptibility of the bulk. Here, we have used the fact that the finite-size corrections to the densities of states in the absence of the Kondo impurity are of $O\left(1 / L^{2}\right)$ implying that all the contributions $O(1 / L)$ are due to the potential scattering in the charge and the Kondo effect in the spin sector. The Kondo temperature $T_{K}$, playing the role of the Fermi temperature in the local Fermi liquid generated by the impurity [19], can be deduced from the spin part of the impurity specific heat as

$$
T_{K}=\frac{3}{2} \pi n v_{s} \frac{\rho_{s}(\infty)}{\delta \rho_{s}(\infty)},
$$

where $n$ is the density of electrons in the system.

The low-temperature thermodynamics is that of a local Fermi liquid, in agreement with one of the two alternatives permitted by conformal field theory. [8] It may perhaps surprise that our results also fall into the framework of the Furusaki-Nagaosa analysis of thermodynamics. [1] To see this, notice that the term responsible for the anomalous scaling of the specific heat in their work is generated by tunneling (in order $J^{-1}$ ) across the Kondo impurity. In our case, however, the open boundary conditions exclude such tunneling processes at all energy scales. Putting the respective coupling constant to zero will produce an excess specific heat linear in temperature, as we have found. This also suggests that the two alternatives given by conformal field theory [B] could, in fact, be just reflect one generic type of scaling behavior and be connected by simply varying this effective tunneling matrix element.

Our finding of local Fermi liquid thermodynamics is a consequence of the LandauLuttinger framework. [18] The structure of the Bethe Ansatz solution guarantees that this picture can indeed be applied. The anomalous scaling of the specific heat cannot be found in such a picture. Whether the corresponding microscopic model can be solved by Bethe Ansatz remains to be seen. Quite generally, the Landau-Luttinger liquid picture can be used to determine the complete changes in the thermodynamics induced by boundaries in integrable quantum systems. Our above determination of the excess specific heat is the first successful application of this idea.

Case (ii): The ground state is described by $N-2$ real $k$ 's and two imaginary $k$ 's at $\pm i c$ and a real set of $\lambda$. The two imaginary $k$ 's correspond two bound states of electrons around the impurity. In the thermodynamic limit, we can again define densities for the real roots as in (8) and their finite size corrections as in (9). They satisfy the set of integral equations

$$
\begin{aligned}
\rho_{c}(k) & =\frac{1}{\pi}+\int_{-\infty}^{\infty} K\left(k-\lambda, \frac{c}{2}\right) \rho_{s}(\lambda) d \lambda, \\
\rho_{s}(\lambda) & =\int_{-k_{0}}^{k_{0}} K\left(\lambda-k, \frac{c}{2}\right) \rho_{c}(k) d k-\int_{-\infty}^{\infty} K\left(\lambda-\lambda^{\prime}, c\right) \rho_{s}\left(\lambda^{\prime}\right) d \lambda^{\prime}, \\
\delta \rho_{c}(k) & =-K\left(k, \frac{c}{2}\right)-2 K(k, c)-\int_{-\infty}^{\infty} K\left(k-\lambda, \frac{c}{2}\right) \delta \rho_{s}(\lambda) d \lambda, \\
\delta \rho_{s}(\lambda) & =2 K\left(\lambda, \frac{3 c}{2}\right)+K(\lambda, c) \\
& +\int_{-k_{0}}^{k_{0}} K\left(\lambda-k, \frac{c}{2}\right) \delta \rho_{c}(k) d k-\int_{-\infty}^{\infty} K\left(\lambda-\lambda^{\prime}, c\right) \delta \rho_{s}\left(\lambda^{\prime}\right) d \lambda^{\prime} .
\end{aligned}
$$

The magnetization at zero temperature can be calculated as above 


$$
M_{s}=\frac{1}{4} \lim _{L \rightarrow \infty}\left\{2 L \int_{-k_{0}}^{k_{0}} \rho_{L}^{c}(k) d k-4 L \int_{-\Lambda}^{\Lambda} \rho_{L}^{s}(\lambda) d \lambda+7\right\}=1
$$

Here, $k_{0}$ is determined from $\int_{-k_{0}}^{k_{0}} \rho_{L}^{c}(k) d k=(N-1 / 2) / L$. We have accounted for the contribution of the two bound electrons in the last term of (19). The ground state now is a spin triplet which apparently violates the Furusaki-Nagaosa conjecture. To understand this phenomenon, recall that the present case corresponds to an attractive potential scattering by the impurity, in addition to ferromagnetic Kondo exchange. Two electrons will then bind to the impurity at $x \approx 0$ and $x \approx L$, and form a spin- $\frac{3}{2}$ complex with the impurity spin. However, the Coulomb interaction is repulsive and induces an indirect antiferromagnetic exchange coupling between the conduction electrons and the $S=3 / 2$-complex. The indirect Kondo coupling between the local composite and the conduction electrons then is equivalent to a spin- $\frac{3}{2}$ single impurity Kondo problem in a Luttinger liquid. Our exact results then indicate that, as in the Fermi liquid, an $S=3 / 2$-impurity is only partially screened [20] in a Luttinger liquid and an effective, unscreened spin-1 results.

The specific heat and the Pauli magnetic susceptibility can also be calculated with the procedure as discussed in case (i). The total susceptibility is divergent at zero temperature due to the nonvanishing moment in the ground state.

The absence of complete Kondo screening and the associated changes in the thermodynamics do not fall into the two universality classes found by conformal field theory. 8] Notice, however, that they are a direct consequence of the attractive potential scattering from the Kondo impurity included in our model in the present case. Our results then imply that new universality classes for the Kondo effect in a Luttinger liquid should exist once potential scattering is included in the conformal field theory.

In summary, we have studied the low energy properties of a ferromagnetic Kondo problem in the $1 \mathrm{D} \delta$-potential Fermi gas. The model Hamiltonian is exactly solvable for two special sets of coupling constants. In one case (repulsive potential scattering off the impurity), the local magnetic moment is completely screened in the ground state which confirms the Furusaki-Nagaosa conjecture, while in the other case (attractive potential scattering), the ground state is a spin triplet. The nonvanishing moment for the latter case is attributed to the formation of the local spin- $\frac{3}{2}$ composite which is partially screened by the Kondo effect due to antiferromagnetic exchange with the remaining conduction electrons. The specific heat and the susceptibility follow the laws of the single channel Kondo system, as a consequence of the strong backscattering [6](open boundary) included in the present model. To the best of our knowledge, this is the first exact solution of the Kondo problem in an interacting many-body system.

The authors acknowledge support from SFB 279 of Deutsche Forschungsgemeinschaft. J. V. is a Heisenberg Fellow of DFG. 


\section{REFERENCES}

[1] F.D.M. Haldane, J. Phys. C 14, 2585 (1981).

[2] For a review, see, e.g., J. Voit, Rep. Prog. Phys. 58, 977 (1995).

[3] D.-H. Lee and J. Toner, Phys. Rev. Lett. 69, 3378 (1992).

[4] A. Furusaki and N. Nagaosa, Phys. Rev. Lett. 72, 892 (1994).

[5] Y. Wang, Chin. Phys. Lett., Sept. 1996 (in press).

[6] M. Fabrizio and A.O. Gogolin, Phys. Rev. B 51, 17827 (1995).

[7] A. Schiller and K. Ingersent, Phys. Rev. B 51, 4676 (1995).

[8] P. Fröjdh and H. Johannesson, Phys. Rev. Lett. 75, 300 (1995).

[9] C. N. Yang, Phys. Rev. Lett. 19, 1312 (1967).

[10] C.L. Kane and M.P.A. Fisher, Phys. Rev. Lett. 68, 1220 (1992); Phys. Rev. B 46, 15233 (1992).

[11] H. Schulz, J. Phys. C18, 581 (1985).

[12] A. Foerster and M. Karowski, Nucl. Phys. B 396, 611 (1993); 408, 512 (1993).

[13] Y. Wang, J. Voit, and F.-C. Pu, Phys. Rev. B 54, Sept. 15, 1996; cond-mat/9602086.

[14] H. Asakawa and M. Suzuki, J. Phys. A: Math. Gen. 29, 225 (1996).

[15] F. C. Alcaraz, M. N. Barber, M. T. Batchelor, R. J. Baxter, and G. R. W. Quispel, J. Phys. A: Math. Gen. 20, 6397 (1987).

[16] F. Woynarovich, Phys. Lett. 108A, 401 (1985).

[17] F. Woynarovich, J. Phys. A: Math. Gen. 22, 4243 (1989).

[18] J. Carmelo and A. A. Ovchinnikov, J. Phys.: Condens. Matter 3, 757 (1991); J. Carmelo, P. Horsch, P. A. Bares, and A. A. Ovchinnikov, Phys. Rev. B 44, 9967 (1991).

[19] P. Nozières, J. Low Temp. Phys. 17, 31 (1974).

[20] N. Andrei, K. Furuya and J. Lowenstein, Rev. Mod. Phys. 55, 331(1983). 